\title{
La criopreservación embrionaria en estadios más avanzados de desarrollo disminuye la frecuencia de pacientes con embriones criopreservados y el número de embriones criopreservados
}

\author{
JUAN ENRIQUE SCHWARZEE ${ }^{1,2, a}$, JOSÉ BALMACEDA ${ }^{1}$, RICARDO POMMER $^{1}$
}

\section{Cryopreservation in blastocyst stage effectively reduce the number of embryos cryopreserved}

Background: Multiple pregnancies are the main complication associated to assisted reproduction, due to the transfer of more than one embryo. Embryo cryopreservation allows the sequential transfer of all generated embryos, thus diminishing the risk of multiple pregnancies. However, it leads to accumulation of cryopreserved embryos. To reduce their accumulation in our unit, we started to preserve embryos as blastocysts, that have a rate of successful pregnancies of approximately 40\%. Aim: To perform a sensitivity analysis of this change of policy on the accumulation of embryos. Material and Methods: Records of 571 cycles of in vitro fertilization since 2007 were reviewed, assuming a transference rate of two embryos in women aged less than 35 years and three embryos in older women. The number of embryos that would be preserved as zygotes, eight cell stage or blastocysts, was analyzed. Results: Multiple component logistic regression analysis showed a 20\% reduction in the odds ratio of cryopreservation per year of age. There was a 95\% reduction in the ratio, when comparing the preservation of third and first day embryos and a $99 \%$ reduction when comparing preservation of embryos in blastocyst stage and first day embryos. Conclusions: Cryopreservation at blastocyst stage effectively decreased the frequency of embryo cryopreservation.

(Rev Med Chile 2012; 140: 45-49).

Key words: Cryopreservation; Embryo; Fertilization.
'Unidad de Medicina Reproductiva de Clínica Monteblanco. Santiago, Chile.

${ }^{2}$ Departamento Clínico de Obstetricia y Ginecología, Universidad de Santiago de Chile.

${ }^{\mathrm{a} M S C}$.

Recibido el 25 de marzo de 2011, aceptado el 17 de octubre de 2011.

Correspondencia a: Dr. Juan Enrique Schwarze; Camino a Farellones 18780 Lo Barnechea, Santiago Chile. Teléfono: 321 5000, E-mail: jeschwarze@ivf.cl
$\mathrm{L}$ a complicación principal de las técnicas de reproducción asistida, como la fertilización in vitro, es la multigestación ${ }^{1}$. Esta se asocia a un aumento importante en la frecuencia de prematurez y aumento de la morbi-mortalidad perinatal. La frecuencia de multigestación en las parejas sometidas a técnicas de reproducción asistida es consecuencia directa de la transferencia de más de un embrión. La transferencia de más de un embrión es un reflejo del deseo de mejorar el resultado del ciclo, ya que al transferir más de un embrión aumentan las posibilidades que al menos uno de ellos sea normal, se implante y origine un embarazo ${ }^{2}$.

La criopreservación embrionaria permitiría la transferencia secuencial y diferida de todos los embriones generados durante un ciclo de reproducción asistida. De este modo, independientemente del número de embriones generados, se transfieren dos o tres -de acuerdo a la morfología embrionaria y edad de la mujer ${ }^{3,4}$ - por intento, disminuyendo significativamente el riesgo de 
multigestación extrema sin afectar la tasa de embarazo acumulado ${ }^{5}$.

Esta técnica se describió en 1983 por Trounson, quien publicó el caso de un embarazo luego de transferencia de un embrión de 8 células congelado/descongelado, que terminó en un parto pretérmino por infección ovular por rotura prematura de membranas ${ }^{6}$. En Chile, el primer caso exitoso de embarazo clínico tras la transferencia de embriones criopreservados fue en 1999; desde entonces el número de ciclos de transferencia de embriones criopreservados ha aumentado considerablemente en nuestro país ${ }^{2}$.

A pesar de todas las ventajas asociadas, este programa tiene algunos inconvenientes, siendo el más importante la acumulación de embriones. Algunas parejas que consiguen un embarazo y el nacimiento de un neonato -especialmente en el caso de partos múltiples-, posponen indiscriminadamente la transferencia de los embriones criopreservados. Muchas parejas tienen varios embriones criopreservados pero con mínimo potencial implantatorio, ya que los mejores embriones de la cohorte ya fueron transferidos. De este modo, tienen que ser sometidas a varios ciclos de transferencia embrionaria, posponiendo el inicio de un nuevo ciclo y aumentando los niveles de estrés y ansiedad en la pareja.

En la actualidad se puede criopreservar embriones en distintos estadios de desarrollo embrionario: zygoto, ocho células y blastocisto, con un distinto potencial implantatorio ocurrido por la selección embrionaria al progresar a estadios más avanzados de desarrollo ${ }^{1}$. También se puede criopreservar ovocitos, en el caso que las pacientes presenten alguna objeción a la criopreservación embrionaria. Recientemente, presentamos el primer caso de nacimiento tras la transferencia de embriones obtenidos por la fecundación de ovocitos vitrificados ${ }^{7}$.

En nuestra unidad, considerando que la transferencia de embriones criopreservados en estadio de blastocisto se asocia a una tasa de embarazo clínico cercana al 40\%, decidimos en el año 2007 criopreservar embriones que alcanzaran estadio de blastocisto, ya sea en el día 5 ó 6 de cultivo in vitro.

El objetivo del presente estudio es analizar la repercusión que tuvo este cambio de política en la proporción de parejas que criopreservaron embriones y el número de embriones criopreservados.

\section{Material y Método}

Revisamos todos los casos de fertilización in vitro (con o sin inyección intracitoplasmática de espermatozoides) entre el 1 de enero de 2007 y el 30 de junio de 2010, e hicimos un análisis de sensibilidad, asumiendo la transferencia de un máximo de dos embriones en mujeres menores de 35 años, y un máximo de tres embriones en mujeres con 35 y más años ${ }^{3,4}$. Analizamos la proporción de pacientes y la mediana de embriones que se criopreservarían con distintas alternativas de criopreservación embrionaria: zigoto, estadio de 8-células, estadio de blastocisto.

Los datos fueron analizados mediante el programa Stata 11 (Statacorp, EEUU) y se presenta los resultados como mediana y rango intercuartílico, y odds ratios con intervalo de confianza de 95\%, cuando corresponda. Hicimos una regresión de Poisson para medidas repetidas para determinar la frecuencia de embriones para criopreservación, ajustada por edad de la mujer y día de cultivo in vitro: 1,3 y 5 .

\section{Resultados}

Analizamos 571 casos consecutivos de ciclos de fertilización in vitro (con o sin inyección intracitoplasmática de espermatozoides) en los que al menos fecundó un ovocito.

La mediana de edad de las mujeres fue 35 (rango intercuartílico 38-32 años), la mediana de ovocitos recuperados fue 9 (rango intercuartílico 14-5); y la mediana del número de ovocitos inseminados fue 6 (rango intercuartílico 15-3). La tasa de fecundación fue $70 \%$, mientras que la tasa global de embarazo clínico por transferencia fue $42 \%$. Las principales características de los ciclos se presentan en la Tabla 1.

Para este estudio, asumimos que en mujeres menores que 35 años se transfirió un máximo de dos embriones, mientras que en mujeres con 35 y más años se transfirió un máximo de tres embriones.

La Tabla 2 muestra los resultados del análisis de sensibilidad, en que se muestra la frecuencia de parejas que criopreservarían embriones de acuerdo al estadio de desarrollo embrionario en el que se criopreservan los embriones. A medida que avanza el estadio elegido para criopreservar desde 
Criopreservación embrionaria - J. E.Schwarze et al

Tabla 1. Características de la población

\begin{tabular}{|c|c|}
\hline Número & 571 \\
\hline Edad Mediana, años (p25 p75) & $35(32-38)$ \\
\hline ICSI (\%) & 90 \\
\hline Mediana ovocitos recuperados (p25-p75) & $9(5-14)$ \\
\hline Mediana embriones transferidos (p25-p75) & $2(1-2)$ \\
\hline Tasa embarazo & $42 \%$ \\
\hline Mediana embriones criopreservados (p25-p75) & $1(0-3)$ \\
\hline $\begin{array}{l}\text { Mediana embriones criopreservados en las } \\
\text { pacientes que criopreservaron ( } \mathrm{p} 25-\mathrm{p} 75)\end{array}$ & $3(2-4)$ \\
\hline
\end{tabular}

zigoto hasta blastocisto, disminuiría el número de zigotos y embriones criopreservados. El 18\% de la cohorte inicial se queda sin embriones para criopreservar, pero evidentemente el $56 \%$ que lo logra dispone de embriones de mejor capacidad funcional. Además, se muestra cómo se estrecha el rango intercuartílico alrededor de la mediana cuando se progresa en el mismo sentido, a pesar de que ésta permanece en 3.

La Tabla 3 muestra el mismo análisis, pero categorizado por edad de la mujer. La edad es un factor pronóstico importante para la criopreservación embrionaria. Las mujeres más jóvenes criopreservarían más frecuentemente en todos los escenarios de criopreservación analizados, y la frecuencia disminuye según progresa el período de cultivo in vitro. La mediana de embriones criopreservados es similar en los grupos de mujeres más jóvenes, pero menor en el grupo de mujeres $\geq 40$ años.

La Tabla 4 muestra el resultado del análisis de regresión de Poisson con componentes mixtos para el número de embriones que se criopreservarían según el día de desarrollo in vitro, corregido por la edad de la mujer. Se puede ver que un aumento unitario en la edad de la mujer disminuye en $8 \%$ el número de embriones disponibles para congelar. La congelación es 20\% menos frecuente en el día 3 respecto del día 1 y $28 \%$ menos frecuente en el día 5 respecto del día 3.

Tabla 2. Frecuencia de parejas que criopreservarían y número de embriones criopreservados de acuerdo al estadio de desarrollo embrionario

\begin{tabular}{|lccc|}
\hline & \multicolumn{2}{c|}{ Estadio de desarrollo embrionario } \\
\hline Parejas que criopreservarían \% (n/total) & Zigoto & 8-células & Blastocisto \\
\hline Mediana embriones criopreservados $(\mathrm{p} 25-\mathrm{p} 75)$ & $74(421 / 571)$ & $63(358 / 571)$ & $56(317 / 571)$ \\
\hline
\end{tabular}

Tabla 3. Frecuencia de parejas que criopreservarían y número de embriones criopreservados de acuerdo al estadio de desarrollo embrionario en que se criopreserva, de acuerdo a la edad de la mujer

\begin{tabular}{|c|c|c|c|}
\hline \multirow[b]{2}{*}{$\leq 34 n=248$} & \multicolumn{3}{|c|}{ Estadio de desarrollo embrionario } \\
\hline & Zigoto & 8-células & Blastocisto \\
\hline Parejas que criopreservarían N (\%) & $195(79)$ & $192(77)$ & $149(60)$ \\
\hline Mediana embriones criopreservados (p25-p75) & $4(2-7)$ & $3(2-6)$ & $3(2-5)$ \\
\hline $35-39 n=230$ & Zigoto & 8-células & Blastocisto \\
\hline Parejas que criopreservarían n (\%) & $170(74)$ & $134(58)$ & $133(58)$ \\
\hline Mediana embriones criopreservados (p25-p75) & $4(1-5)$ & $3(2-5)$ & $2(2-4)$ \\
\hline$\geq 40=93$ & Zigoto & 8-células & Blastocisto \\
\hline Parejas que criopreservarían n (\%) & $56(60)$ & $32(34)$ & $30(32)$ \\
\hline Mediana embriones criopreservados (p25-p75) & $1(1-3)$ & $2(0-3)$ & $2(2-4)$ \\
\hline
\end{tabular}


Tabla 4. Razón de Índice de Incidencia para embriones criopreservados obtenidos mediante análisis de regresión de Poisson para medidas repetidas

\begin{tabular}{|lccc|}
\hline Factor & IRR & $\begin{array}{c}\text { Intervalo confianza } \\
\mathbf{9 5 \%}\end{array}$ & p \\
\hline Edad & 0,92 & $0,89-0,95$ & 0,001 \\
\hline D3 vs D1 & 0,80 & $0,74-0,86$ & 0,001 \\
\hline D5 vs D3 & 0,72 & $0,66-0,78$ & 0,001 \\
\hline
\end{tabular}

Tabla 5. Odds ratio para criopreservación embrionaria obtenidos mediante análisis de regresión logística mixto

\begin{tabular}{|lccc|}
\hline Factor & OR & $\begin{array}{c}\text { Intervalo confianza } \\
\mathbf{9 5 \%}\end{array}$ & p \\
\hline Edad & 0,81 & $0,71-0,91$ & 0,001 \\
\hline D3 vs D1 & 0,05 & $0,02-0,09$ & 0,001 \\
\hline D5 vs D1 & 0,01 & $0,00-0,02$ & 0,001 \\
\hline
\end{tabular}

Finalmente, para determinar la probabilidad que una pareja criopreserve embriones en función de la edad de la mujer y el estadio de desarrollo embrionario, hicimos un análisis de regresión logística con componentes mixtos. Los resultados se muestran en la Tabla 5. Se ve una disminución de $20 \%$ en la odds ratio por cada año de la paciente, y una disminución de $95 \%$ al comparar la probabilidad de criopreservar el tercer día de desarrollo in vitro versus el primer día; y de $99 \%$ al comparar el quinto día con el primer día.

\section{Discusión}

Actualmente, los resultados de las técnicas de reproducción asistida en Latinoamérica son comparables a los de los países desarrollados. Este éxito se ha logrado, en parte, transfiriendo un mayor número de embriones. En efecto, en el año 2009 se transfirió una mediana de 2,4 embriones en ciclos de fecundación in vitro, y en $44 \%$ de las transferencias se transfirió tres o más embriones. Esto se traduce en una frecuencia de parto doble de $21,9 \%$ y de parto triple o mayor de $2,1 \%{ }^{8,9}$ [ZegersHochschild 2008a ]. Por lo tanto, uno de los grandes desafíos es reducir la frecuencia de multigestación ${ }^{10}$. Es por eso que las sociedades médicas de países desarrollados abogan por la transferencia de uno o dos embriones, de acuerdo a la edad de la paciente y los resultados anteriores ${ }^{11-16}$.

La reducción en el número de embriones transferidos sin sacrificar la tasa de embarazo acumulado sólo se puede lograr con la criopreservación y posterior transferencia de los embriones no transferidos, lo que puede redundar en la acumulación de embriones. Presentamos un análisis de sensibilidad que muestra una disminución significativa en la proporción de parejas que criopreservan según se pospone el estadio de desarrollo embrionario para la criopreservación. embrionaria. Junto con el estadio de desarrollo embrionario al criopreservar embriones, encontramos que la edad de la paciente es un importante factor predictivo. Esto coincide con los resultados publicados por el último Registro Latinoamericano de Reproducción Asistida ${ }^{1}$.

Es interesante constatar que la cantidad de embriones criopreservados no difirió significativamente entre los distintos grupos. Finalmente, los embriones con más días de cultivo in vitro tienen mejor tasa de implantación, por lo que la criopreservación en este estadio se asocia a una mejor tasa de embarazo ${ }^{1}$, lo que supone un nuevo beneficio para las parejas.

De acuerdo al último registro latinoamericano de reproducción asistida, sólo 15\% de las transferencias de embriones criopreservados son realizadas en estadio de blastocisto ${ }^{1}$, por lo que creemos que reportes como el nuestro van a ayudar a cambiar esa conducta y a reducir el número de embriones criopreservados.

\section{Referencias}

1. Zegers-Hochschild F, Schwarze JE, Galdames V. XIX Registro Latinoamericano de Reproduccion Asistida 2008. 2010.

2. Schwarze JE, Galdames V. Reproducción asistida en Latinoamérica y en Chile. Rev Med Clin Las Condes. 2010; 21 (3): 451-6.

3. JOINT SOGC-CFAS. Guidelines for the number of embryos to transfer following in vitro fertilization No. 182, September 2006. Int J Gynaecol Obstet 2008; 102 (2): 203-16

4. Min JK, Claman P, Hughes E, Society of Obstetricians 
and Gynecologists of Canada, and Canadian Fertility and Andrology Society. Guidelines for the number of embryos to transfer following in vitro fertilization. J Obstet Gynaecol Can 2006; 28 (9): 799-813.

5. Veleva Z, Karinen P, Tomás C, Tapanainen JS, Martikainen $H$. Elective single embryo transfer with cryopreservation improves the outcome and diminishes the costs of IVF/ICSI. Hum Reprod 2009; 24(7): 1632-9.

6. Trounson A, Mohr L. Human pregnancy following cryopreservation, thawing and transfer of an eight-cell embryo. Nature 1983; 305 (5936): 707-9.

7. International Committee for Monitoring Assisted Reproductive Technology, de Mouzon J, Lancaster P, Nygren KG, Sullivan E, Zegers-Hochschild F, et al. World collaborative report on Assisted Reproductive Technology, 2002. Hum Reprod 2009; 24 (9): 2310-20.

8. Zegers-Hochschild F, Schwarze JE, Galdames V. Assisted reproductive technology in Latin America: an example of regional cooperation and development. ESHRE Monographs issue:ESHRE Special Task Force on 'Developing Countries and Infertility’ 2008; 142-47.

9. Gurgan T, Demirol A. Why and how should multiple pregnancies be prevented in assisted reproduction treatment programmes? Reprod Biomed Online 2004; 9 (2): 237-44.

10. Bontekoe S, Blake D, Heineman MJ, Williams EC,
Johnson N. Adherence compounds in embryo transfer media for assisted reproductive technologies. Cochrane Database Syst Rev 2010; 7CD007421.

11. Jungheim ES, Ryan GL, Levens ED, Cunningham AF, Macones GA, Carson KR, et al. Embryo transfer practices in the United States: a survey of clinics registered with the Society for Assisted Reproductive Technology. Fertil Steril 2010; 94 (4) :1432-6.

12. Cutting R, Morroll D, Roberts SA, Pickering S, Rutherford A, and BFS and ACE. Elective single embryo transfer: guidelines for practice British Fertility Society and Association of Clinical Embryologists. Hum Fertil (Camb) 2008; 11 (3): 131-46.

13. Stern JE, Cedars MI, Jain T, Klein NA, Beaird CM, Grainger DA, et al. Assisted reproductive technology practice patterns and the impact of embryo transfer guidelines in the United States. Fertil Steril 2007; 88 (2): 275-82.

14. Gibbons W, Grainger D, Cedars M, Jain T, Klein N, Stern J, and SART Research Committee Writing Group. Continuous quality improvement and assisted reproductive technology multiple gestations: some progress, some answers, more questions. Fertil Steril 2007; 88 (2): 301-4.

15. Jain T, Missmer SA, Hornstein MD. Trends in embryotransfer practice and in outcomes of the use of assisted reproductive technology in the United States. N Engl J Med 2004; 350 (16): 1639-45. 